\title{
3Photochemistry of Sulfilimine-based Nitrene Precursors: Generation of Both Singlet and Triplet Benzoylnitrene
}

\author{
Vasumathi Desikan, ${ }^{\dagger}$ Yonglin Liu, ${ }^{\ddagger}$, John P. Toscano, ${ }^{\ddagger, *}$ and William S. Jenks ${ }^{\dagger, *}$ \\ ${ }^{\dagger}$ Department of Chemistry, Iowa State University, 3760 Gilman Hall, Ames, IA 50011, \\ ${ }^{*}$ Department of Chemistry, Johns Hopkins University, 3400 North Charles Street, Baltimore, MD
} 21218

wsjenks@iastate.edu

jtoscano@jhu.edu

\section{Supporting Information}

Table of Contents

Spectral Data for compounds

S2

Kinetic Traces for IR Data

S7

Energies and Coordinates for Computed Structures

$\mathrm{S} 10$

Tabulated Calculated Vibrational Frequencies

$\mathrm{S} 12$ 
N-benzoyldibenzothiophene sulfilimine (2)

(Prepared by Method A)

${ }^{1} \mathrm{H}$ NMR (400 MHz, $\mathrm{CDCl}_{3}$ )

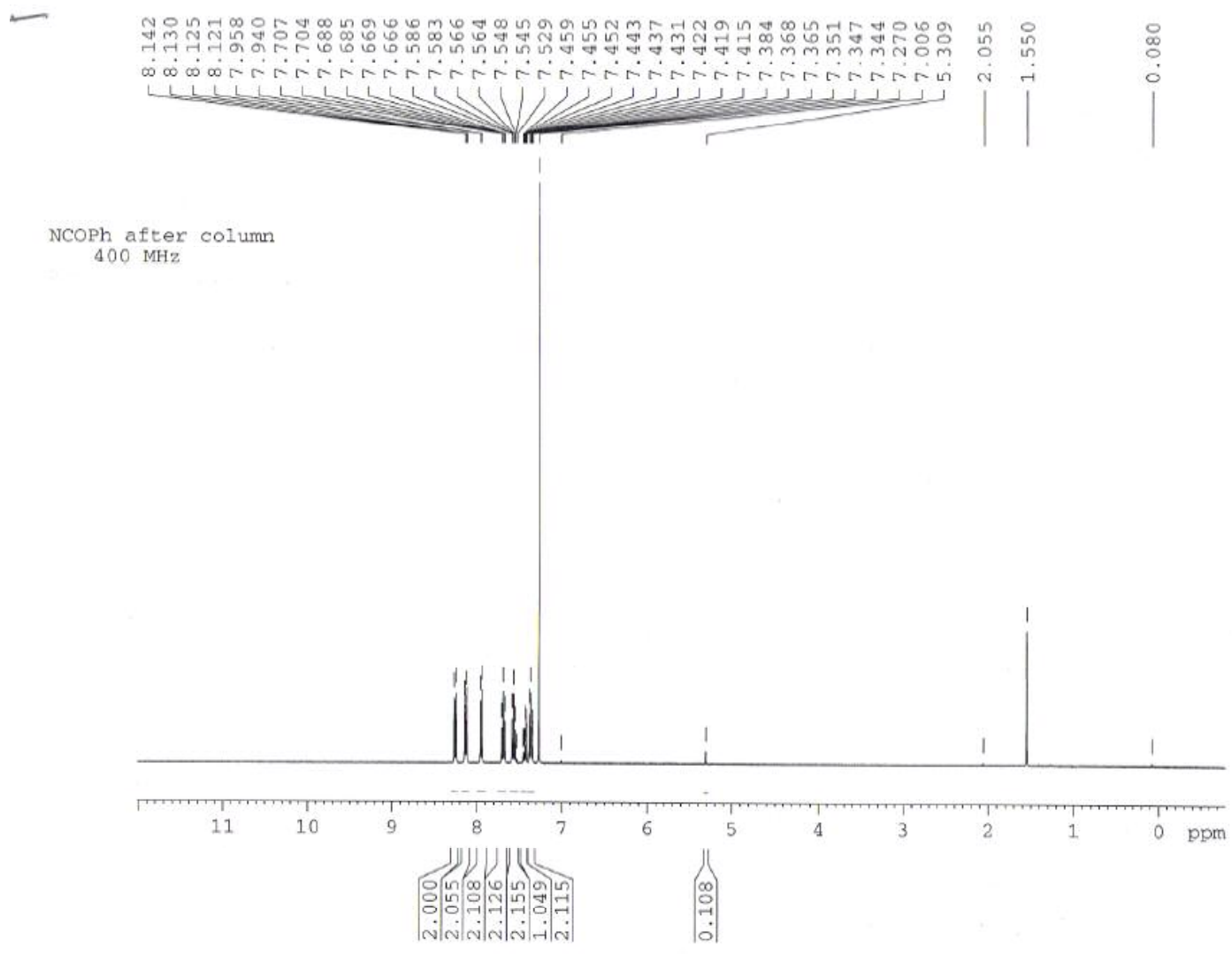




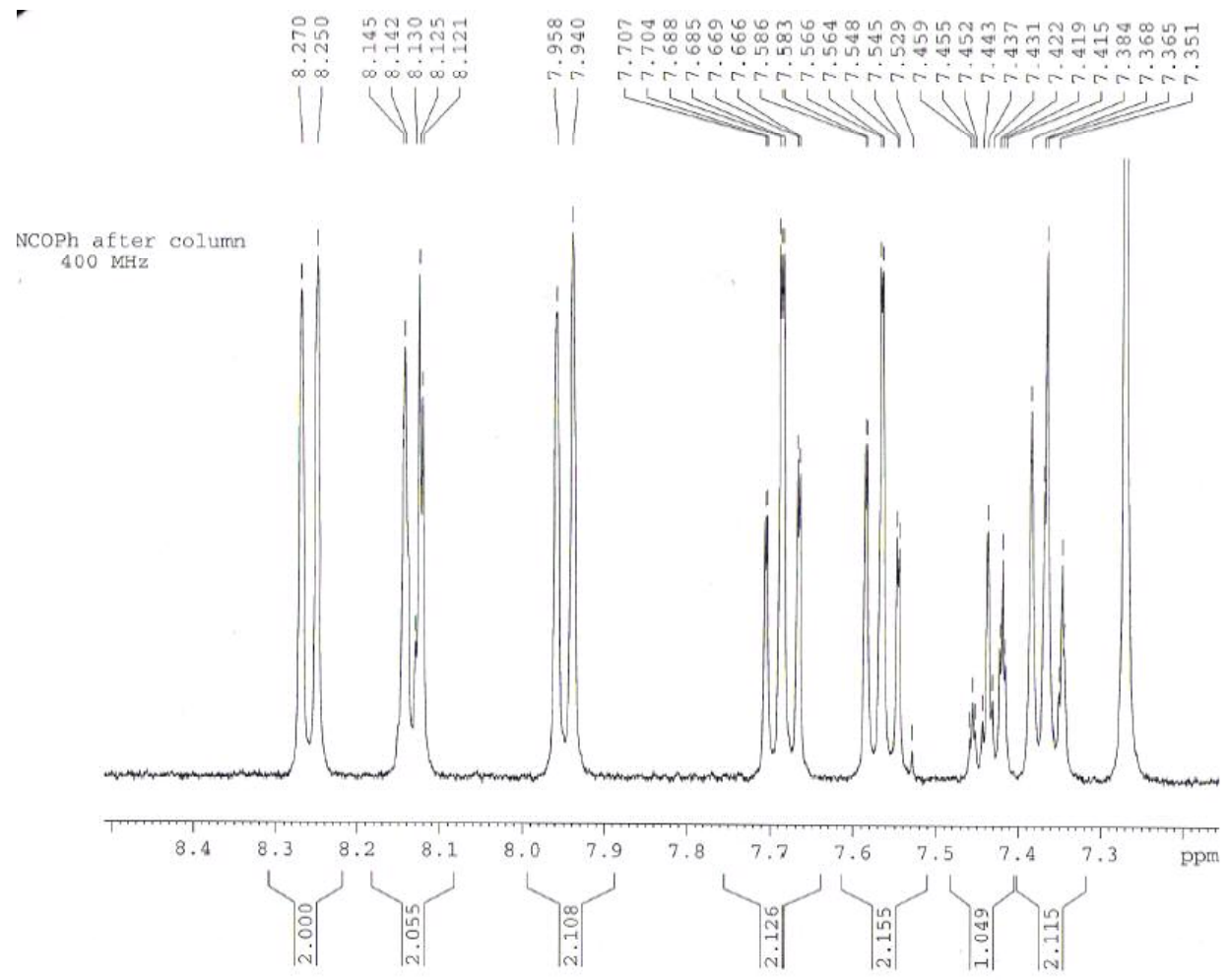

${ }^{13} \mathrm{C} \mathrm{NMR}\left(100 \mathrm{MHz}, \mathrm{CDCl}_{3}\right)$
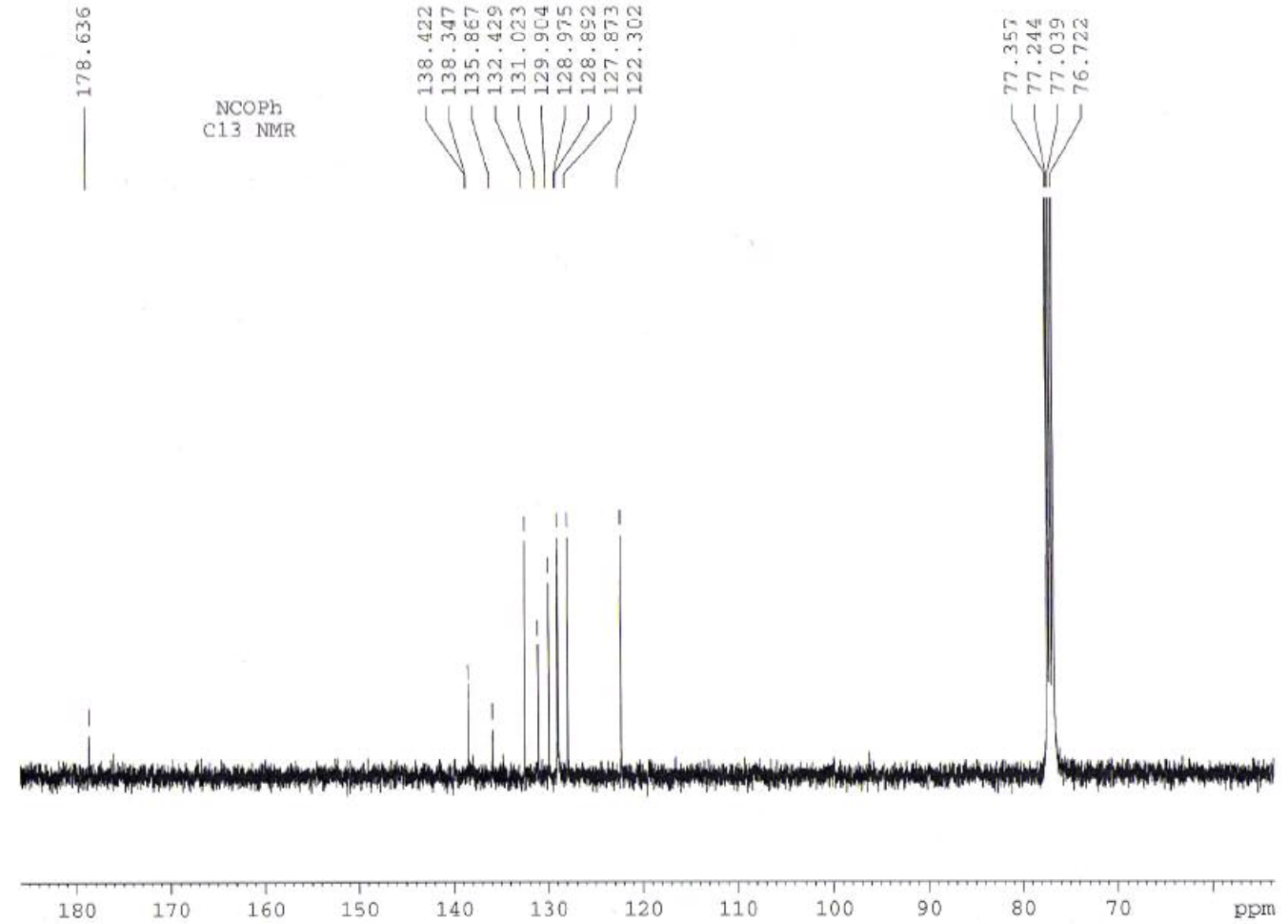


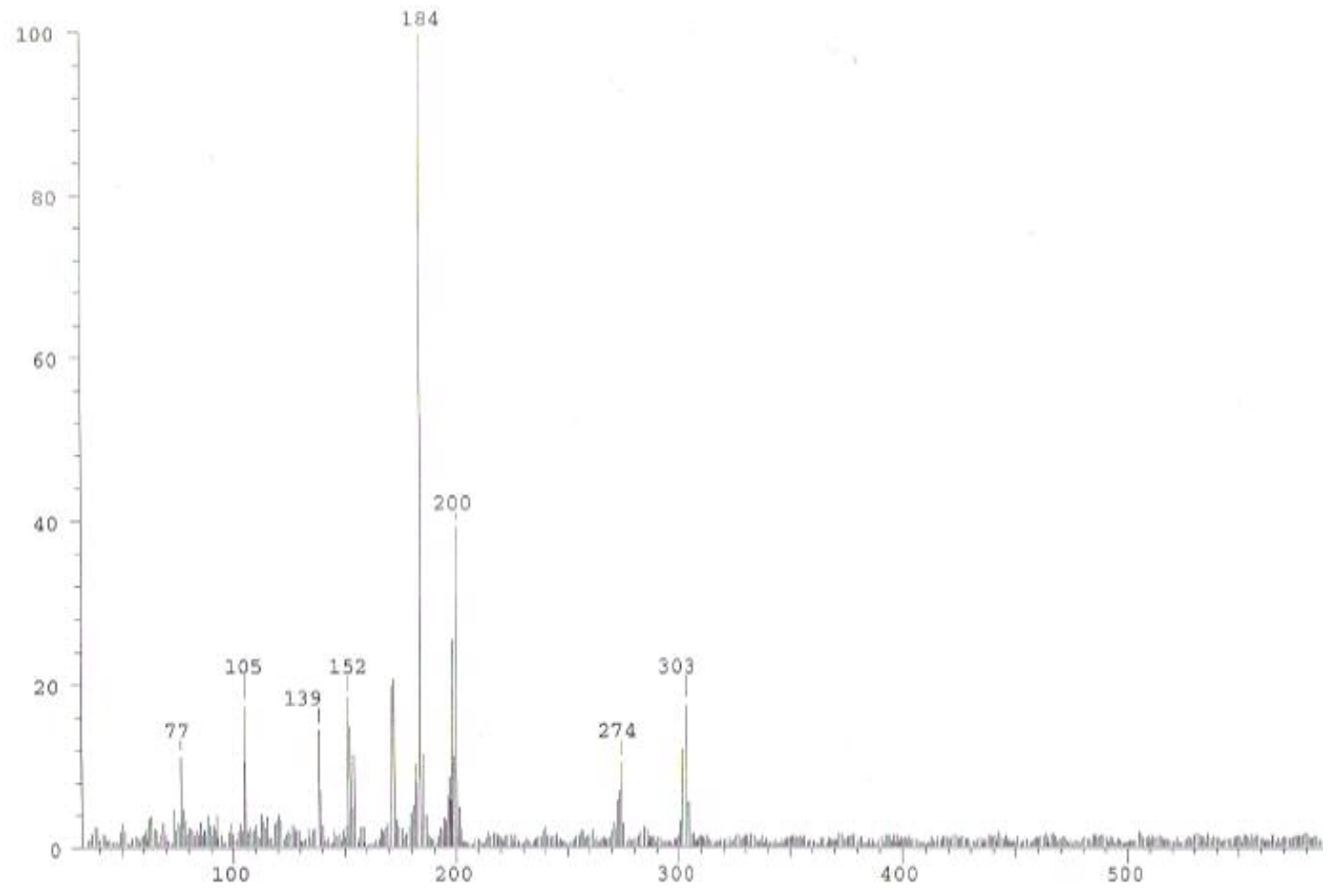




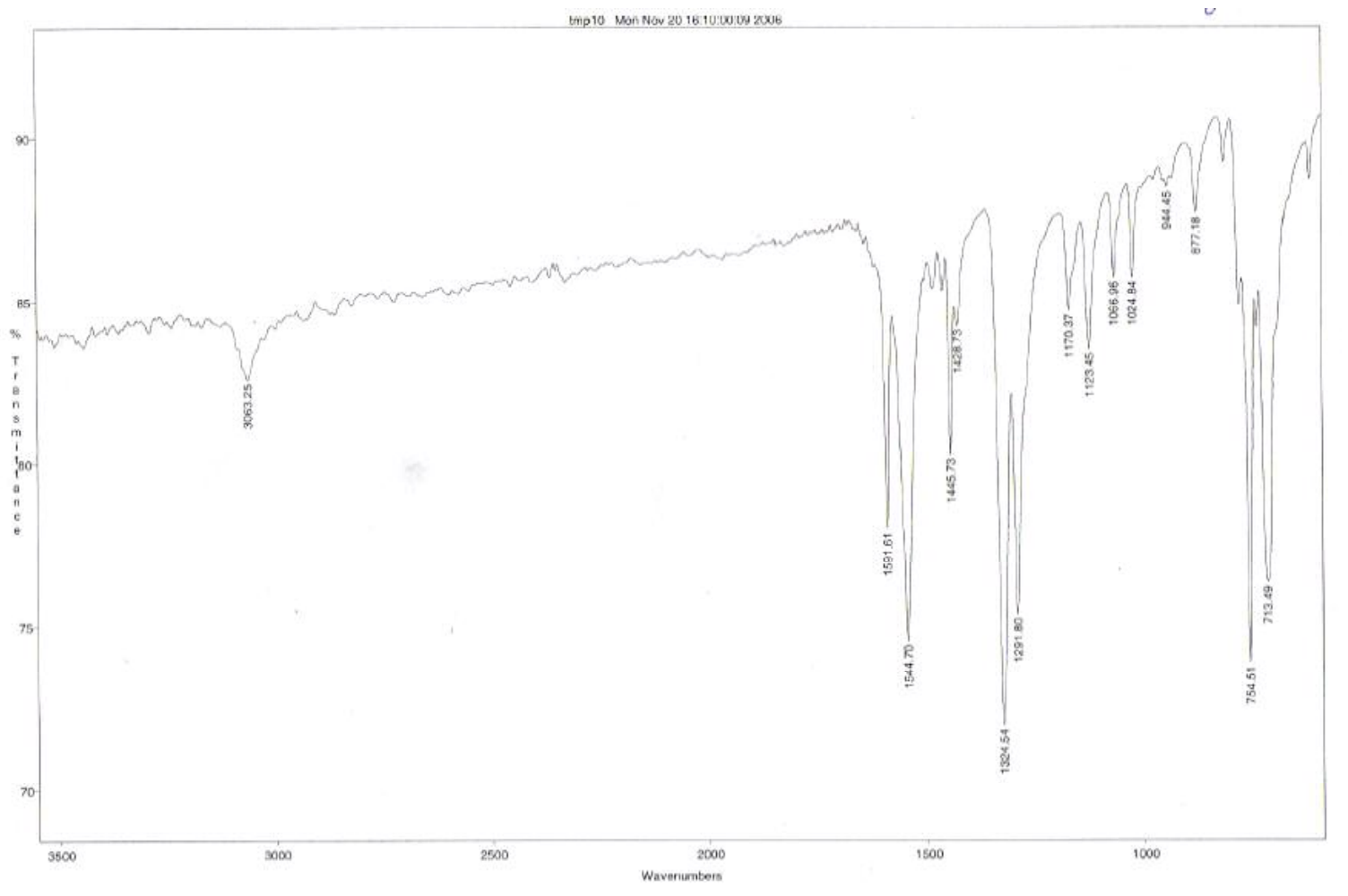




\section{Threo $N$-benzoyl-2,3-di-n-propyl azridine (12a)}

${ }^{1} \mathrm{H}$ NMR $\left(400 \mathrm{MHz}, \mathrm{CDCl}_{3}\right)$

요욤

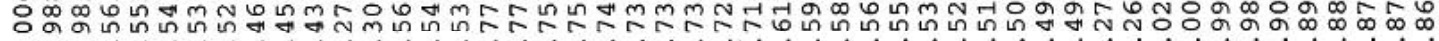

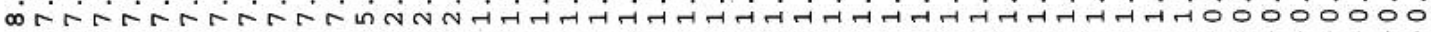



NCOPh cis aziridine

after column

$400 \mathrm{MHz}$

vasu-01-08-07-003

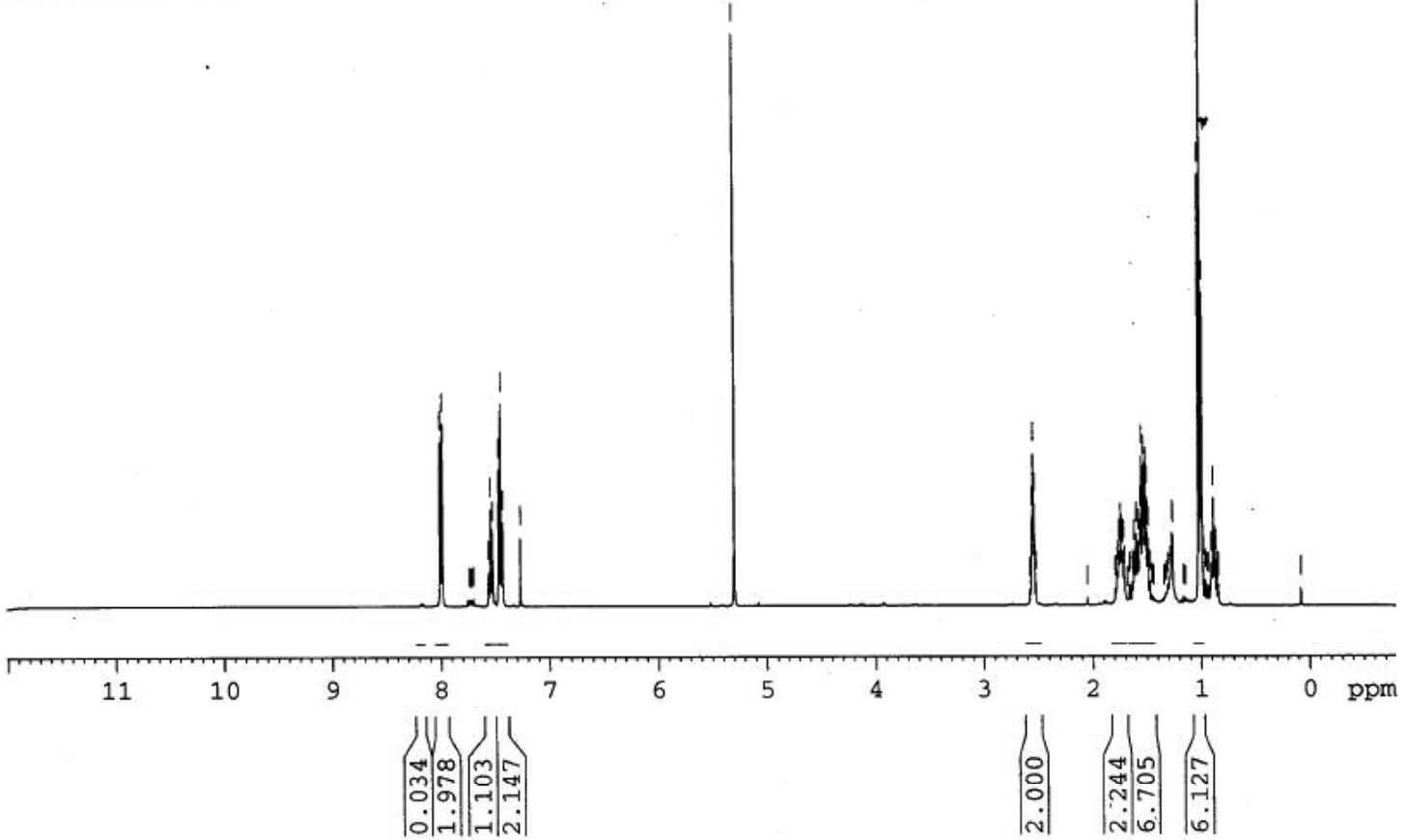




\section{${ }^{13} \mathrm{C}$ NMR $\left(100 \mathrm{MHz}, \mathrm{CDCl}_{3}\right)$}

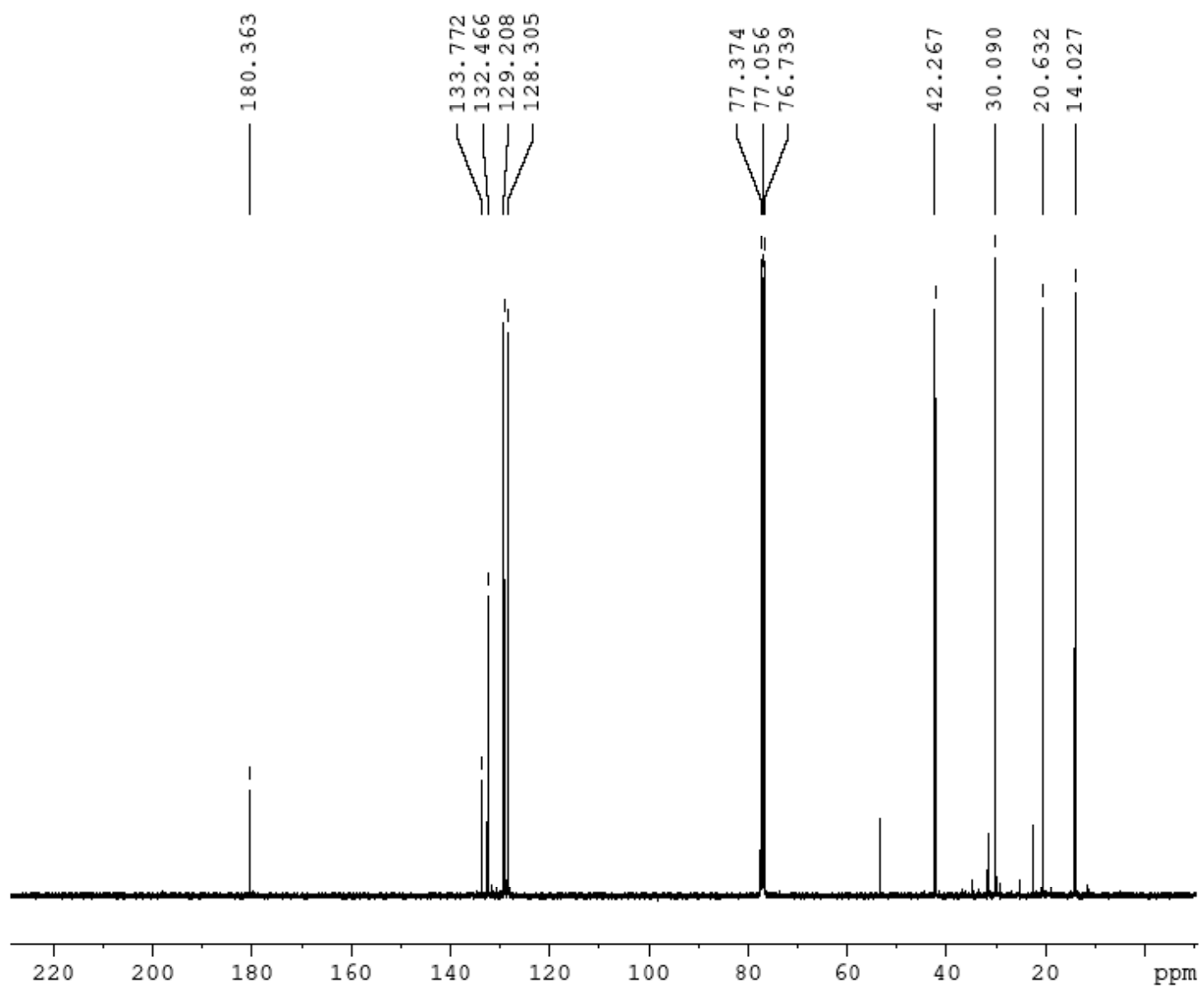



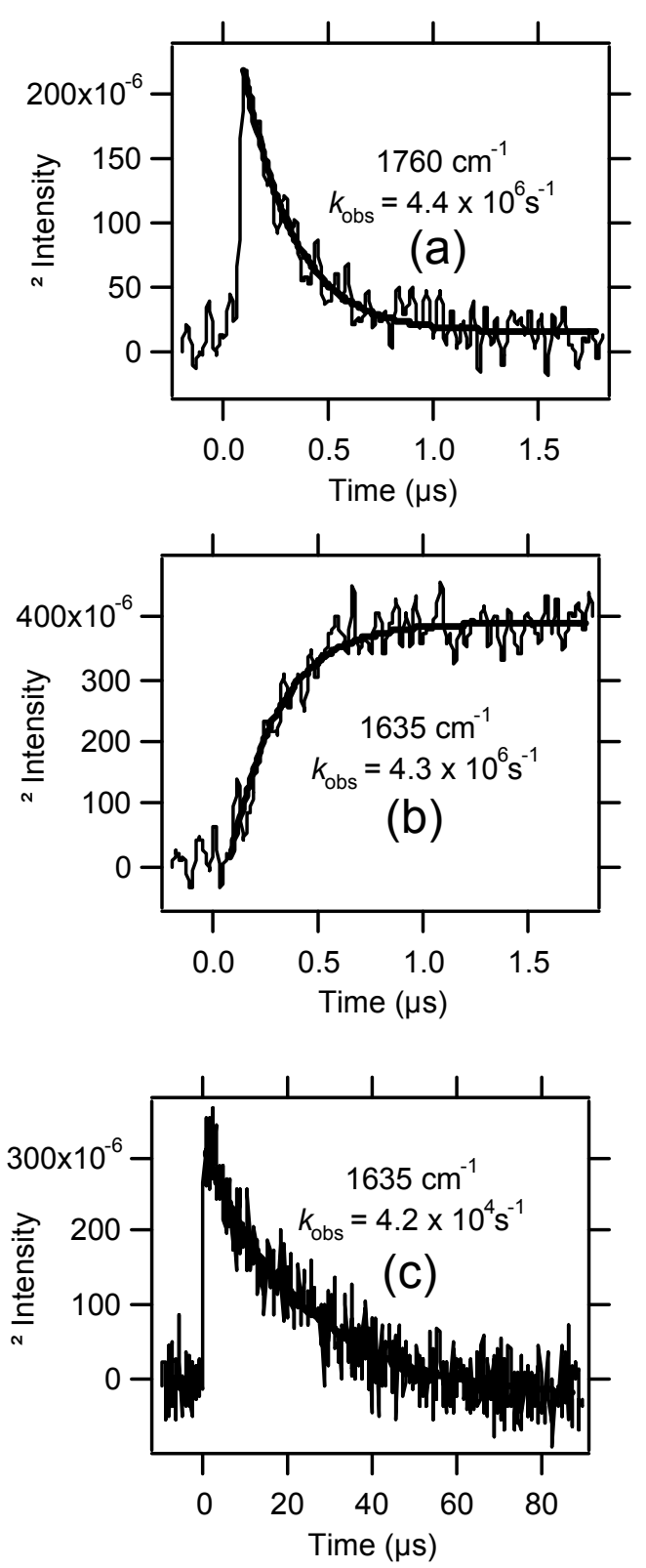

Figure S1. Kinetic traces observed at (a) $1760 \mathrm{~cm}^{-1}$, (b) $1635 \mathrm{~cm}^{-1}$ from -0.2 to $1.8 \mu \mathrm{s}$, and (c) $1635 \mathrm{~cm}^{-1}$ from -10 to $90 \mu$ s following $266 \mathrm{~nm}$ laser photolysis of sulfilimine 1 in argon-purged acetonitrile- $d_{3}$. The dotted-line curves are experimental data and the solid lines are calculated best fits to a single-exponential function. 

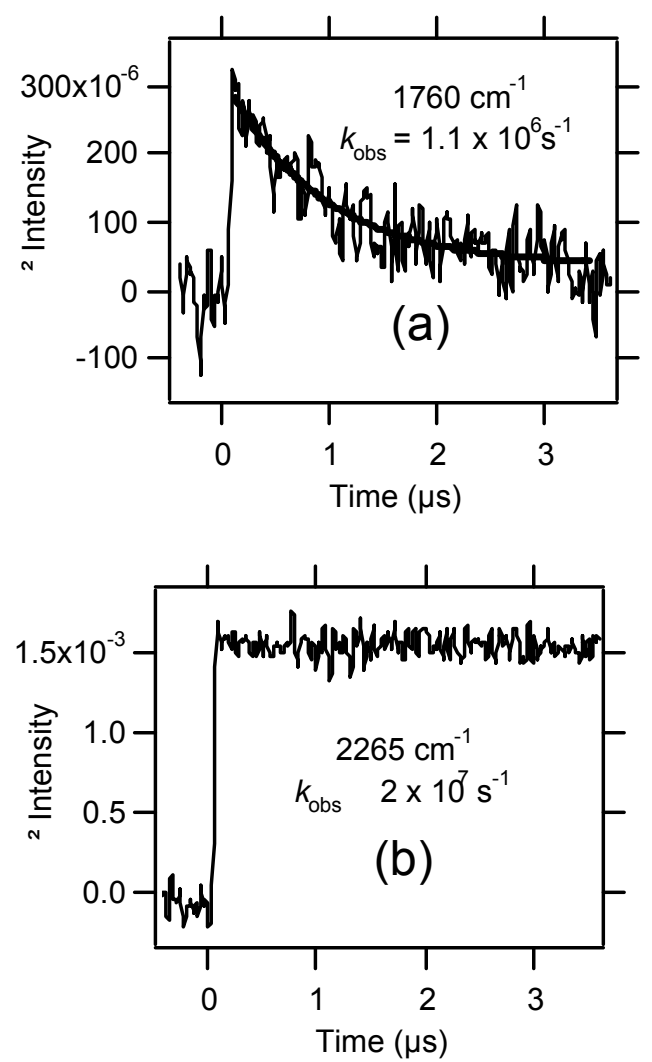

Figure S2. Kinetic traces observed at (a) 1760 and (b) $2265 \mathrm{~cm}^{-1}$ following $266 \mathrm{~nm}$ laser photolysis of sulfilimine $1(1 \mathrm{mM})$ in argon-purged dichloromethane. The dotted-line curves are experimental data and the solid lines are calculated best fits to a single-exponential function. 

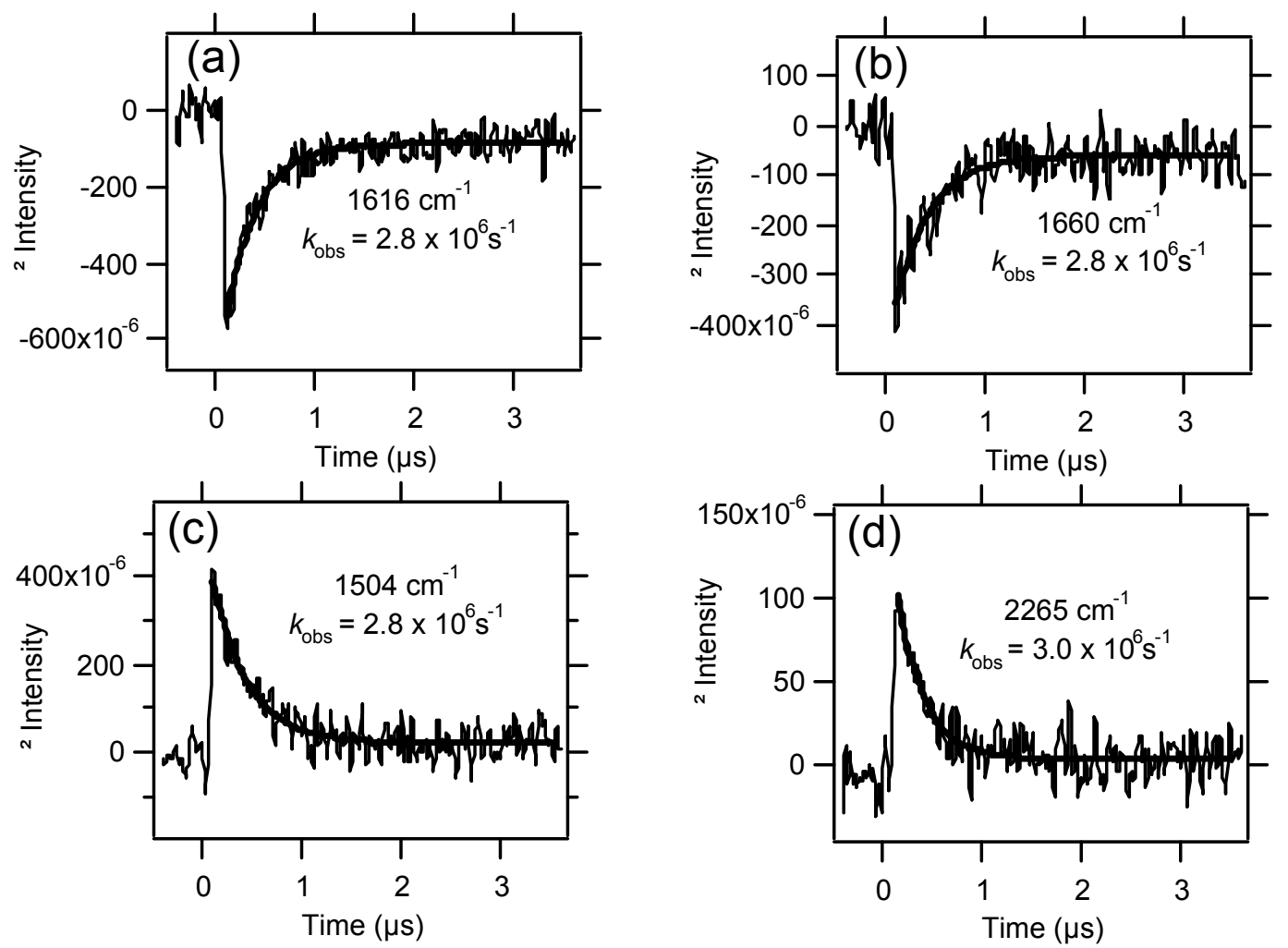

Figure S3. Kinetic traces observed at (a) 1616, (b) 1660, (c) 1504, and (d) $2265 \mathrm{~cm}^{-1}$ following $355 \mathrm{~nm}$ laser photolysis of xanthone $(5 \mathrm{mM})$ in argon-saturated acetonitrile- $d_{3}$. The dotted curves are experimental data; the solid curves are the calculated best fit to a single-exponential function. 
Table 1. Optimized geometry and energy for singlet benzoylnitrene ${ }^{\mathbf{1}}$.

Theory: B3LYP/6-31G*

Zero-point correction $=$

Thermal correction to Energy=

.102293 (Hartree/Particle)

Thermal correction to Enthalpy=

.109502

Thermal correction to Gibbs Free Energy=

.110446

Sum of electronic and zero-point Energies $=-399.510643$

Cartesian Coordinates:

\begin{tabular}{lcrl}
\hline Atomic & \multicolumn{2}{l}{ Coordinates (Angstroms) } \\
Number & $\mathrm{X}$ & $\mathrm{Y}$ & $\mathrm{Z}$ \\
\hline 8 & 2.593343 & -0.855067 & -0.000007 \\
7 & 2.669690 & 0.903945 & 0.000000 \\
6 & -1.791882 & -1.243026 & 0.000000 \\
6 & -2.537806 & -0.061352 & -0.000003 \\
6 & -1.897173 & 1.182374 & -0.000002 \\
6 & -0.507264 & 1.248270 & 0.000001 \\
6 & 0.238879 & 0.060559 & 0.000004 \\
6 & -0.400070 & -1.186242 & 0.000004 \\
6 & 1.689314 & 0.101889 & 0.000006 \\
1 & -2.295110 & -2.205384 & 0.000000 \\
1 & -3.623330 & -0.108354 & -0.000006 \\
1 & -2.482881 & 2.096848 & -0.000005 \\
1 & 0.003761 & 2.206971 & 0.000001 \\
1 & 0.198999 & -2.091990 & 0.000006 \\
\hline
\end{tabular}


Table 2. Optimized geometry and energy for triplet benzoylnitrene ${ }^{\mathbf{3}} \mathbf{4}$.

Theory: B3LYP/6-31G*

Zero-point correction $=$

Thermal correction to Energy=

.101584 (Hartree/Particle)

Thermal correction to Enthalpy=

.108705

.109649

Thermal correction to Gibbs Free Energy $=\quad .068475$

Sum of electronic and zero-point Energies $=-399.519307$

Cartesian Coordinates:

\begin{tabular}{lrrr}
\hline Atomic & \multicolumn{2}{l}{ Coordinates (Angstroms) } \\
Number & $\mathrm{X}$ & $\mathrm{Y}$ & $\mathrm{Z}$ \\
\hline 8 & 2.467403 & -1.036208 & -0.000028 \\
7 & 2.473928 & 1.188780 & 0.000033 \\
6 & -1.789528 & -1.218366 & 0.000012 \\
6 & -2.496656 & -0.010903 & 0.000000 \\
6 & -1.811867 & 1.206611 & -0.000012 \\
6 & -0.418422 & 1.221121 & -0.000011 \\
6 & 0.292510 & 0.011166 & 0.000000 \\
6 & -0.398331 & -1.209646 & 0.000011 \\
6 & 1.776438 & -0.003704 & -0.000002 \\
1 & -2.326051 & -2.162920 & 0.000022 \\
1 & -3.583277 & -0.020350 & 0.000000 \\
1 & -2.363115 & 2.142496 & -0.000021 \\
1 & 0.119458 & 2.164807 & -0.000019 \\
1 & 0.171394 & -2.133500 & 0.000019 \\
\hline
\end{tabular}


Table 3. Optimized geometry and energy for triplet excited state of azide $\mathbf{3}$.

Theory: B3LYP/6-31G*

Zero-point correction=

Thermal correction to Energy=

.109550 (Hartree/Particle)

Thermal correction to Enthalpy=

.118943

Thermal correction to Gibbs Free Energy=

.119887

Sum of electronic and zero-point Energies $=-508.940923$

Cartesian Coordinates:

\begin{tabular}{llrl}
\hline Atomic & \multicolumn{3}{l}{ Coordinates (Angstroms) } \\
Number & $\mathrm{X}$ & $\mathrm{Y}$ & $\mathrm{Z}$ \\
\hline 8 & -1.003036 & 2.306399 & -0.292072 \\
7 & -2.247069 & 0.403770 & -0.266456 \\
7 & -2.480357 & -0.713127 & 0.234887 \\
7 & -2.848074 & -1.746317 & 0.569020 \\
6 & 1.755917 & -1.554398 & -0.384202 \\
6 & 2.857918 & -0.791519 & 0.055288 \\
6 & 2.669545 & 0.549724 & 0.417190 \\
6 & 1.411806 & 1.125006 & 0.361879 \\
6 & 0.280742 & 0.358410 & -0.076132 \\
6 & 0.495795 & -0.996969 & -0.475782 \\
6 & -0.993619 & 0.988328 & -0.139279 \\
1 & 1.901066 & -2.592827 & -0.669621 \\
1 & 3.843807 & -1.242556 & 0.111188 \\
1 & 3.513384 & 1.141779 & 0.760599 \\
1 & 1.254949 & 2.151313 & 0.673307 \\
1 & -0.329051 & -1.590679 & -0.854827 \\
\hline
\end{tabular}


Table 4. B3LYP/6-31G* calculated frequencies $\left(\mathrm{cm}^{-1}\right.$, unscaled) and intensities for singlet benzoylnitrene ${ }^{\mathbf{1}} \mathbf{4}$.

\begin{tabular}{lc|lc}
\hline Frequency & Intensity & Frequency & Intensity \\
\hline 74 & 0.0 & 1052 & 2.0 \\
145 & 2.2 & 1109 & 8.0 \\
155 & 3.4 & 1181 & 10.0 \\
360 & 2.7 & 1198 & 0.3 \\
411 & 0.8 & 1212 & 1.8 \\
413 & 1.0 & 1280 & 27.1 \\
504 & 0.0 & 1363 & 4.0 \\
554 & 15.9 & 1372 & 5.9 \\
608 & 29.9 & 1501 & 16.5 \\
630 & 0.1 & 1541 & 6.4 \\
700 & 27.2 & 1643 & 0.9 \\
774 & 0.5 & 1662 & 8.4 \\
781 & 25.6 & 1820 & 187.7 \\
863 & 0.0 & 3192 & 0.1 \\
948 & 1.5 & 3201 & 2.6 \\
979 & 0.0 & 3207 & 9.8 \\
1011 & 0.0 & 3216 & 12.1 \\
1019 & 0.4 & 3221 & 8.4 \\
\hline
\end{tabular}


Table 5. B3LYP/6-31G* calculated frequencies $\left(\mathrm{cm}^{-1}\right.$, unscaled) and intensities for triplet benzoylnitrene ${ }^{3} 4$.

\begin{tabular}{lc|lc}
\hline Frequency & Intensity & Frequency & Intensity \\
\hline 66 & 0.0 & 1051 & 5.1 \\
156 & 1.0 & 1083 & 14.6 \\
197 & 1.6 & 1117 & 3.6 \\
353 & 5.2 & 1196 & 0.9 \\
412 & 0.1 & 1207 & 27.4 \\
434 & 0.8 & 1243 & 97.0 \\
478 & 1.5 & 1356 & 9.4 \\
572 & 32.0 & 1370 & 5.9 \\
629 & 0.1 & 1497 & 21.9 \\
650 & 35.2 & 1533 & 30.8 \\
706 & 43.1 & 1560 & 114.0 \\
760 & 1.9 & 1640 & 0.4 \\
795 & 10.5 & 1657 & 33.9 \\
867 & 0.0 & 3190 & 0.1 \\
954 & 1.1 & 3199 & 6.1 \\
986 & 0.1 & 3206 & 14.6 \\
1012 & 0.0 & 3215 & 13.3 \\
1018 & 1.0 & 3223 & 5.6 \\
\hline
\end{tabular}


Table 6. B3LYP/6-31G* calculated frequencies $\left(\mathrm{cm}^{-1}\right.$, unscaled) and intensities for the lowest triplet excited state of benzoyl azide $\mathbf{3}$.

\begin{tabular}{lc|lr}
\hline Frequency & Intensity & Frequency & Intensity \\
\hline 50 & 0.0 & 965 & 59.5 \\
88 & 1.4 & 969 & 0.5 \\
107 & 0.7 & 990 & 0.0 \\
113 & 0.1 & 996 & 88.4 \\
218 & 0.1 & 1115 & 14.9 \\
308 & 0.0 & 1205 & 3.9 \\
320 & 4.0 & 1250 & 6.5 \\
378 & 0.0 & 1301 & 111.3 \\
406 & 1.3 & 1347 & 192.9 \\
470 & 3.7 & 1359 & 6.2 \\
508 & 8.9 & 1394 & 5.7 \\
510 & 0.4 & 1419 & 63.1 \\
546 & 1.4 & 1453 & 92.6 \\
588 & 60.6 & 1589 & 11.1 \\
643 & 1.1 & 1687 & 47.6 \\
697 & 0.0 & 2279 & 1067.8 \\
739 & 0.0 & 3201 & 6.6 \\
796 & 29.0 & 3205 & 13.4 \\
830 & 25.9 & 3231 & 6.1 \\
890 & 49.1 & 3243 & 2.1 \\
895 & 4.7 & 3256 & 1.4 \\
\hline
\end{tabular}

